\title{
miR-483-3p regulates osteogenic differentiation of bone marrow mesenchymal stem cells by targeting STAT1
}

\author{
YE XIAO $^{1}$, QI GUO ${ }^{1}$, TIE-JIAN JIANG ${ }^{1}$, YING YUAN $^{1}$, LI YANG $^{1}$, \\ GUANG-WEI WANG $^{2}$ and WEN-FENG XIAO ${ }^{3}$ \\ ${ }^{1}$ Department of Endocrinology, Endocrinology Research Center, Central South University, Changsha, \\ Hunan 410008; ${ }^{2}$ Department of Medicine, Hunan University of Medicine, Huaihua, Hunan 418000; \\ ${ }^{3}$ Department of Orthopaedics, Xiangya Hospital, Central South University, Changsha, Hunan 410008, P.R. China
}

Received October 30, 2018; Accepted May 15, 2019

DOI: $10.3892 / \mathrm{mmr} .2019 .10700$

\begin{abstract}
Osteogenic differentiation of bone marrow mesenchymal stem cells (BMSCs) is regulated by a variety of intracellular regulatory factors including osterix, runt-related transcription factor 2 (RUNX2), bone morphogenetic proteins and transforming growth factor $\beta$. Recent studies have shown that microRNAs (miRs) serve a crucial role in this process. In the present study, miR-483-3p levels were significantly increased during osteogenic differentiation of mouse and human BMSCs. Overexpression of miR-483-3p promoted osteogenic differentiation, whereas inhibition of miR-483-3p reversed these effects. miR-483-3p regulated osteogenic differentiation of BMSCs by targeting STAT1, and thus enhancing RUNX2 transcriptional activity and RUNX2 nuclear translocation. In vivo, overexpression of miR-483-3p using a BMSC-specific aptamer delivery system stimulated bone formation in aged mice. Therefore, the present study suggested that miR-483-3p promoted osteogenic differentiation of BMSCs by targeting STAT1, and miR-483-3 prepresent a potential therapeutic target for age-related bone loss.
\end{abstract}

\section{Introduction}

Bone remodeling is finely regulated by the balance between bone formation and bone resorption (1). In adult organisms, bone formation is mediated by the recruitment of bone marrow mesenchymal stem cells (BMSCs), which can differentiate into osteoblast cells $(2,3)$. A previous study identified that inhibition of osteogenic differentiation of BMSCs leads to impaired bone formation and contributes to osteoporosis (4). Therefore, to treat bone loss, it is necessary to understand the regulatory

Correspondence to: Professor Wen-Feng Xiao, Department of Orthopaedics, Xiangya Hospital, Central South University, 87 Xiangya Road, Changsha, Hunan 410008, P.R. China

E-mail: xiaowf11@sina.com

Key words: osteogenic differentiation, microRNA-483-3p, STAT1 mechanisms underlying osteogenic differentiation of BMSCs in order to identify novel potential therapeutic targets.

MicroRNAs (miRNAs) are single-stranded small RNA molecules of $\sim 22$ nucleotides in length that regulate translation of target mRNAs by binding to their 3'untranslated region (UTR) and mediating translational inhibition or degradation (5). Previous studies have demonstrated that miRNAs have essential roles in a variety of biological processes, including cellular proliferation, apoptosis, differentiation and immunity (6-8). Numerous previous studies identified various miRNAs able to regulate osteogenic differentiation by targeting important osteogenic factors, such as histone deacetylase 9 (HDAC9), RPTOR independent companion of MTOR complex 2, HDAC5 and runt-related transcription factor 2 (RUNX2) $(4,9,10,11)$. However, the roles and the number of miRNAs involved in osteogenic differentiation remain unclear.

In the present study, miR-483-3p was identified to be significantly upregulated during osteogenic differentiation of BMSCs. Overexpression of miR-438-3p using agomiR-438-3p markedly promoted osteogenic differentiation in vitro, whereas inhibition of miR-438-3p using antagomiR-438-3p reversed these effects. Moreover, BMSC-targeted aptamer-agomiR-483-3p enhanced bone formation in vivo. Collectively, the present study identified a novel role for miR-483-3p and its underlying mechanism in promoting osteogenic differentiation of BMSCs.

\section{Materials and methods}

Animals and reagents. In total, 20 female C57BL/6J mice of 12 months of age and weighing 35-40 g were purchased from Hunan Slaccas Jingda Laboratory Animal Co., Ltd. All mice were maintained in the specific pathogen-free facility of The Laboratory Animal Research Center at Central South University. Animals were housed under a controlled temperature $\left(25 \pm 1^{\circ} \mathrm{C}\right)$ and humidity (50\%), with a 12-h light/dark cycle and free access to food and water. All animal experimental procedures were approved by The Animal Care and Use Committee of The Xiangya Hospital of Central South University. AgomiR-negative control (NC), agomiR-438-3p, antagomiR-NC and antagomiR-483-3P were purchased 
from Shanghai GenePharma Co., Ltd. The BMSC-specific aptamer (5'-GAATTCAGTCGGACAGCGACGACGGTG ATATGTCAAGGTCGTATGCACGAGTCAGAGGGATGG ACGAATATCGTCTCCC-3') was synthesized by GenScript Corporation.

Cell transfection. BMSCs were seeded into 6-well plates $\left(1 \times 10^{6}\right.$ cells/well) and cultured at $37^{\circ} \mathrm{C}$ for $24 \mathrm{~h}$. After $24 \mathrm{~h}$, BMSCs were transfected with $100 \mathrm{nM}$ agomiR-NC (5'-UUU GUACUACACAAAAGUACUG-3'), agomiR-438-3p (5'-UCA CUCCUCCCCUCCCGUCUU-3'), antagomiR-NC (5'-CAG UACUUUUGUGUAGUACAAA-3') or antagomiR-483-3P (5'-A AG ACG GGA GGG GAGGAGUGA-3') using Lipofectamine ${ }^{\circledR} 2000$ (Invitrogen; Thermo Fisher Scientific, Inc.) $48 \mathrm{~h}$ prior to further experimentation, according to the manufacturer's protocol.

BMSC culture and osteogenic differentiation. Mouse BMSCs were isolated from bone marrow of femurs and tibia of C57BL/6J mice (age, 6-8 weeks) and cultured in $\alpha$-MEM medium containing 10\% FBS (Gibco; Thermo Fisher Scientific, Inc.), $1 \%$ penicillin and streptomycin (Gibco; Thermo Fisher Scientific, Inc.) at $37^{\circ} \mathrm{C}$ with $5 \% \mathrm{CO}_{2}$, as previously described (4). Human MSCs (hMSCs) were isolated from the bone marrow of 30 healthy donors (female; age, $25.0 \pm 4.0$ years) and the procedure was approved by The Ethics Committee of The Xiangya Hospital of Central South University. All patients provided written informed consent. Osteogenesis induction medium containing 10\% FBS, $300 \mathrm{ng} / \mathrm{ml}$ bone morphogenetic protein 2 (Sigma-Aldrich; Merck KGaA), $50 \mu \mathrm{g} / \mathrm{ml}$ ascorbic acid and $5 \mathrm{mM} \beta$-glycerol phosphate (Sigma-Aldrich; Merck KGaA) was used to induce the osteogenic differentiation of BMSCs and hMSCs for 0, 3, 7,14 and 21 days.

miRNA microarray assay and miRNA targets prediction. Total RNA was extracted from $3 \times 10^{6}$ BMSCs using TRIzol ${ }^{\circledR}$ reagent (Invitrogen; Thermo Fisher Scientific, Inc.) after treatment with osteogenic medium for 7 days. Total RNA was quantified using the NanoDrop ND-2000 (Thermo Fisher Scientific, Inc.) and the RNA integrity was assessed using the Agilent Bioanalyzer 2100 (Agilent Technologies, Inc.). Then, total RNA was dephosphorylated, denatured and labeled with Cyanine-3-CTP using the miRNA Complete Labeling and Hub kit (Agilent Technologies, Inc.), according to the manufacturer's protocol. The labeled RNAs were hybridized onto a mouse miRNA $8 \times 15 \mathrm{~K}$ chip (Agilent Technologies, Inc.). After washing, all slides were scanned with the Agilent Scanner G2505C (Agilent Technologies, Inc.). Feature Extraction software (version 10.7.1.1; Agilent Technologies, Inc.) and GeneSpring software (version 13.1; Agilent Technologies, Inc.) were used to analyze the microarray results. Differentially expressed miRNAs were then identified based on the fold change and $\mathrm{P}<0.05$, calculated using Student's t-test, as previously described (4). In total, 4 experimental groups (control group and osteogenic differentiation group) were analyzed, $\mathrm{n}=5 / \mathrm{group}$. The target genes of miRNAs were predicted using TargetScan Human version 7.2 (http://www.targetscan.org) and miRanda (http://www. microrna.org/microrna/home.do).
Alkaline phosphatase (ALP) activity, osteocalcin (OCN) secretion assay and Alizarin Red S Staining. ALP activity was determined using an enzymatic colorimetric ALP kit (Roche Diagnostics), according to the manufacturer's protocol. Secreted levels of OCN were measured using a specific OCN immunoassay kit (cat. no. 310950; DiaSorin SpA), according to the manufacturer's protocol. Following culture with osteogenesis induction medium for 21 days, BMSCs were fixed with $4 \%$ paraformaldehyde at $4^{\circ} \mathrm{C}$ for $10 \mathrm{~min}$ and then stained with 2\% Alizarin Red S (Sigma-Aldrich; Merck KGaA) at room temperature for $5 \mathrm{~min}$ to evaluate cell matrix mineralization. Staining results were visualized using a Diaphot Inverted Microscope and Camera System (magnification, x100; Leica Microsystems $\mathrm{GmbH}$ ), as previously described (12). $\mathrm{n}=5$ in each group.

$R N A$ isolation and reverse transcription quantitative $(R T-q)$ $P C R$. Total RNA was extracted from $3 \times 10^{6}$ BMSCs using TRIzol reagent (Invitrogen; Thermo Fisher Scientific, Inc.). The mRNAs were reverse-transcribed into cDNA using PrimeScript RT reagent kit with gDNA Eraser (Takara Bio, Inc) by incubating at $37^{\circ} \mathrm{C}$ for $30 \mathrm{~min}$. The qPCR was performed using SYBR ${ }^{\circledR}$ Premix Ex Taq ${ }^{\mathrm{TM}}$ (Takara Bio, Inc.) with an ABI 7900 thermocycler (Thermo Fisher Scientific, MA, USA) using 40 cycles of $95^{\circ} \mathrm{C}$ for $5 \mathrm{sec}$ and $60^{\circ} \mathrm{C}$ for $30 \mathrm{sec}$. Nucleotide sequences of primers for STAT1, RUNX2, osterix, Type 1 collagen, ALP, OCN and $\beta$-actin are listed in Table I. miRNAs were reverse-transcribed at $37^{\circ} \mathrm{C}$ for 60 min using One Step PrimeScript ${ }^{\circledR}$ miRNA cDNA Synthesis kit (Takara Bio, Inc.). Primers for HsnRNA U6 (cat. no. HmiRQP9001), MsnRNA U6(cat. no. MmiRQP9002), hsa-miR-483 (cat. no. HmiRQP0517), mmu-miR-483 (cat. no. MmiRQP1052) were purchased from GeneCopoeia, Inc. The qPCR was performed using SYBR ${ }^{\circledR}$ Premix Ex Taq ${ }^{\mathrm{TM}}$ (Takara Bio, Inc.) with an ABI 7900 thermocycler (Thermo Fisher Scientific, Inc) using the following thermocycling conditions: 40 cycles of $95^{\circ} \mathrm{C}$ for $5 \mathrm{sec}$ and $60^{\circ} \mathrm{C}$ for $30 \mathrm{sec}$. The expression levels of the target genes were calculated using the $2^{-\Delta \Delta C q}$ method (13). $\beta$-actin and U6 were used as an internal control for normalizing the expression of mRNA and miRNA, respectively.

Plasmid construction and luciferase reporter assay. The 3'-UTR of STAT1, containing the predicted miR-483-3p binding sites, was amplified by PCR using the Phanta Super-Fidelity DNA Polymerase (Vazyme) from complementary DNA, using the STAT1 forward primer, 5'-GGG GTACCAGCCATAAACTTGGAGAA-3' and STAT1 reverse primer, 5'-CCCAAGCTTTCTTGCCCTGACTTGGAT-3'. The PCR thermocycling conditions were as follows: $95^{\circ} \mathrm{C}$ for $2 \mathrm{~min}$ followed by 30 cycles of $95^{\circ} \mathrm{C}$ for $30 \mathrm{sec}, 55^{\circ} \mathrm{C}$ for $30 \mathrm{sec}$ and $72^{\circ} \mathrm{C}$ for $60 \mathrm{sec}$, and a final extension at $72^{\circ} \mathrm{C}$ for $10 \mathrm{~min}$. The PCR products were digested using KpnI and HindIII, and inserted into apGL3-basic vector (Promega Corporation) to generate the reporter vector wild-type-pGL3-STAT1 3'-UTR. A vector containing a mutant version of the STAT1 3'-UTR was constructed using STAT1-mutant forward primer, 5'-GGGGTACCCTTCCCATGTCTCCAGTTAAGTT-3' and STAT1 reverse primer, 5'-CCCAAGCTTTCTTGCCCT GACTTGGAT-3'. Reporter plasmid containing the promoter 
Table I. Nucleotide sequences of primers used for quantitative PCR.

\begin{tabular}{|c|c|c|}
\hline Gene & Genbank accession no. & Primer sequence $\left(5^{\prime}-3^{\prime}\right)$ \\
\hline RUNX2 & NM_001146038 & $\begin{array}{l}\text { F: AGAGTCAGATTACAGATCCCAGG } \\
\text { R: TGGCTCTTCTTACTGAGAGAGG }\end{array}$ \\
\hline Osterix & NM_130458.4 & $\begin{array}{l}\text { F: ACCAGGTCCAGGCAACAC } \\
\text { R: GCAAAGTCAGATGGGTAAGTAG }\end{array}$ \\
\hline STAT1 & XM_017319463.1 & $\begin{array}{l}\text { F: TCACAGTGGTTCGAGCTTCAG } \\
\text { R:GCAAACGAGACATCATAGGCA }\end{array}$ \\
\hline Type 1 collagen & NM_007742 & $\begin{array}{l}\text { F: GCTCCTCTTAGGGGCCACT } \\
\text { R: CCACGTCTCACCATTGGGG }\end{array}$ \\
\hline ALP & XM_017319924 & $\begin{array}{l}\text { F:CCCCATGTGATGGCGTAT } \\
\text { R:CGGTAGGGAGAGCACAGC }\end{array}$ \\
\hline OCN & NM_001032298 & $\begin{array}{l}\text { F:AAGCAGGAGGGCAATAAGGT } \\
\text { R:ATGCGTTTGTAGGCGGTCTT }\end{array}$ \\
\hline$\beta$-actin & NM_007393.5 & $\begin{array}{l}\text { F: GGCTGTATTCCCCTCCATCG } \\
\text { R: CCAGTTGGTAACAATGCCATGT }\end{array}$ \\
\hline
\end{tabular}

F, forward; R, reverse; RUNX2, runt-related transcription factor 2; ALP, alkaline phosphatase; OCN, osteocalcin.

of OCN was constructed as previously described (14). The RUNX2 or STAT1 coding sequence was cloned into the pCMV-Myc vector (Sino Biological, Inc.) using EcoRI and KpnI restriction enzymes, generatingRUNX2 or STAT1 overexpression plasmids. BMSCs cells were transfected with $2 \mu \mathrm{g}$ of the RUNX2 plasmid or the STAT1 plasmids along with $100 \mathrm{nM}$ of agomiR-483-3P or antagomiR-483-3 Pusing Lipofectamine ${ }^{\circledR}$ 2000. Relative luciferase activities were determined by a dual-luciferase reporter assay system (Promega Corporation). Luciferase activity was determined $48 \mathrm{~h}$ after transfection. Renilla activity was used to normalize Firefly luciferase activity.

Western blot analysis. BMSCs were collected and lysed in RIPA buffer (Beyotime Institute of Biotechnology) with protease inhibitors (Roche Diagnostics) for $30 \mathrm{~min}$ on ice. Cells were then sonicated at a power level of $55 \%$ on a microtip-equipped Sonic Dismembrator for $5 \mathrm{sec}, 3$ times, on ice. After sonication, the cell lysates were cleared by centrifugation at $14,000 \mathrm{x} \mathrm{g}$ for $10 \mathrm{~min}$ at $4^{\circ} \mathrm{C}$. Nuclear protein was isolated using a Nuclear and Cytoplasmic Protein Extraction kit (Beyotime Institute of Biotechnology), according to the manufacturer's instructions (15). The protein concentration was measured using the Coomassie-blue method, and equal amounts of total protein $(15 \mu \mathrm{g} /$ lane $)$ were separated on a $12 \%$ SDS-PAGE, and transferred to PVDF membranes. PVDF membranes were then blocked with $5 \%$ BSA for $1 \mathrm{~h}$ at room temperature and incubated with primary antibodies overnight at $4^{\circ} \mathrm{C}$ : Mouse-anti-Runx2 (1:1,000; cat. no. ab76956; Abcam), rabbit-anti-STAT1 (1:1,000; cat. no. 14994; Cell Signaling Technology, Inc.), rabbit-anti-proliferating cell nuclear antigen (1:1,000; cat. no. 13110; Cell Signaling Technology, Inc.) and mouse-anti- $\beta$-actin (1:2,000; cat. no. BM5422; Boster Biological Technology). The following day, membranes were washed with $0.1 \%$ TBS-Tween 20 (TBST) and incubated with the appropriate horseradish peroxidase-conjugated secondary antibody (1:2,000; cat. nos. sc-2004 and sc-2005; Santa Cruz Biotechnology, Inc.) for $1 \mathrm{~h}$ at room temperature. After washing, protein bands were visualized using SuperSignal West Dura Extended Duration Substrate (Pierce; Thermo Fisher Scientific, Inc.). Western blot analysis was performed in triplicate.

Microcomputed tomography analysis. Female mice (age, 12 months) were randomly divided into three groups $(n=6$ per group). The BMSC-specific aptamer-agomiR-483-3p was synthesized by GenScript Corporation. Aptamer-agomiR-NC, Aptamer-agomiR-483-3P or PBS was periosteally injected into the medullary cavity of the femur twice per month for 3 months. After 3 months of treatment, microcomputed tomography analysis, three-point bending test and bone histomorphometrically analysis were performed as previously reported $(4,16)$.

Statistical analysis. Statistical analysis was performed using SPSS (version 13.0; SPSS, Inc.). Data are presented as the mean \pm SD. One-way ANOVA followed by Dunnett's post hoc test was used to analyze differences among multiple groups. All experiments were repeated $>3$ times. $\mathrm{P}<0.05$ was considered to indicate a statistically significant difference.

\section{Results}

miR-483-3p is increased during osteogenic differentiation. To investigate changes in miRNA expression during osteogenic differentiation, BMSCs were cultured with osteogenic medium for 7 days. Changes in miRNA expression were identified by performing miRNA microarray analysis of differentiated BMSCs compared with undifferentiated control samples. The $\mathrm{z}$-score illustrated the relative expression level 
A

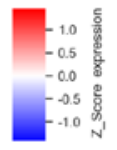

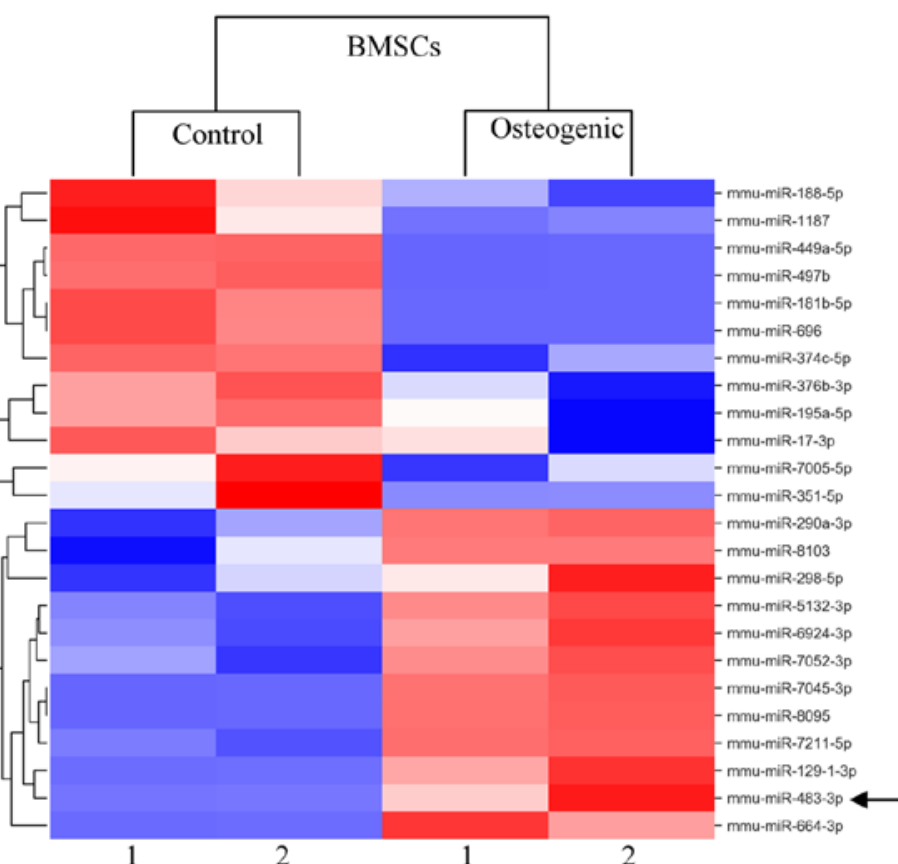

C

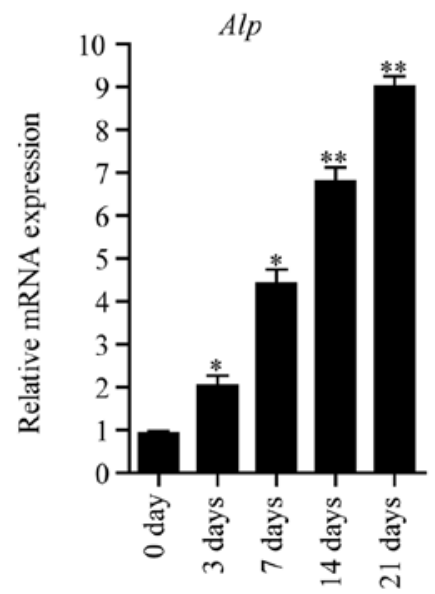

D

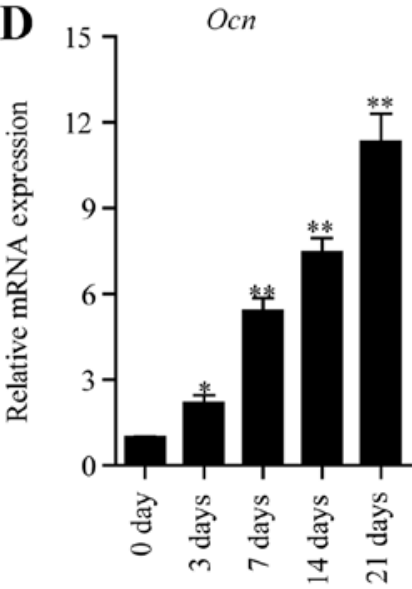

B

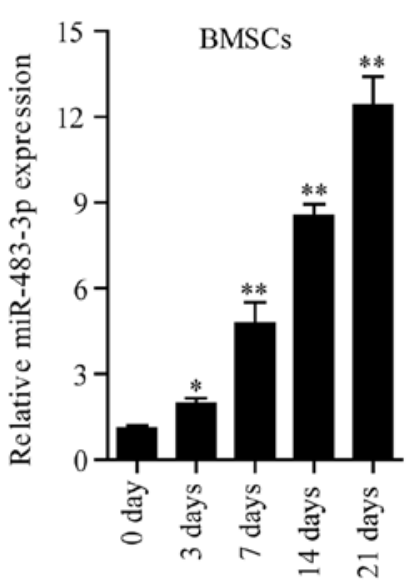

E

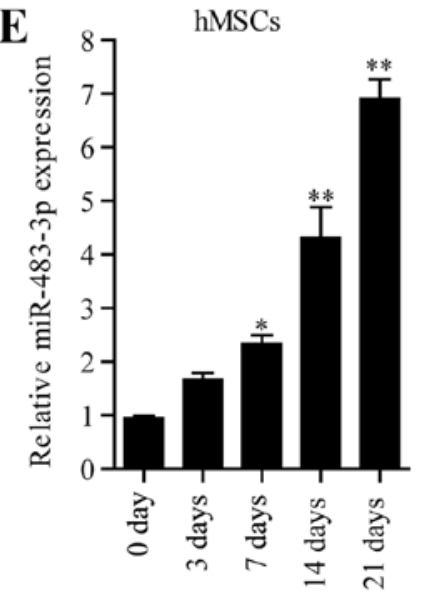

Figure 1. Expression level of miR-483-3p during osteogenic differentiation of BMSCs. (A) Changes in miRNA expression were analyzed by miRNA microarray using BMSCs cultured in osteogenic differentiation medium or in $\alpha$-MEM control medium for 7 days. Red indicates increased expression and blue indicates decreased expression compared with the control group. (B) RT-qPCR analysis of the expression level of miR-483-3p in BMSCs cultured in osteogenic differentiation medium for 0,3, 7, 14 and 21 days. RT-qPCR analysis of the mRNA levels of (C) OCN and (D) ALP at 0, 3, 7, 14 and 21 days. (E) RT-qPCR analysis of the expression level of miR-483-3p in hMSCs cultured in osteogenic differentiation medium for $0,3,7,14$ and 21 days. Data are presented as the mean \pm SD. $\mathrm{n}=5$ per group. ${ }^{*} \mathrm{P}<0.05,{ }^{* *} \mathrm{P}<0.01$ vs. 0 day. BMSCs, bone marrow mesenchymal stem cells; miRNA, microRNA; RT-qPCR, reverse transcription-quantitative PCR; ALP, alkaline phosphatase; OCN, osteocalcin; hMSCs, human mesenchymal stem cells; miR, microRNA.

of a specific miRNA. Each row represents an miRNA and each column represents a sample. From the analysis of the microarray data based on the fold change and probability values, 10 miRNAs were found to be downregulated and 11 were upregulated significantly (fold change, $>2 ; \mathrm{P}<0.05$ ). Certain miRNAs that have previously been reported to regulate osteogenic differentiation, such as miR-129, miR-188, miR-195 and miR-497 (4,17,18), were detected in the present microarray analysis (Fig. 1A). Among the differentially expressed miRNAs identified, miR-483-3p was significantly upregulated during osteogenic differentiation (Fig. 1A). Previous studies have shown that miR-483-3p is involved in cell proliferation, cell cycle and apoptosis $(19,20)$, but its role in osteogenic differentiation remains uninvestigated. To examine the expression level of miR-483 during osteogenic differentiation, BMSCs were treated with osteogenic differentiation medium for $0,3,7,14$, and 21 days, and the expression level of miR-483-3p was assessed by RT-qPCR. The qPCR results suggested that miR-483-3p levels gradually increased during osteogenic differentiation, in line with the miRNA microarray analysis results (Fig. 1B). Additionally, the expression levels of osteogenic gene markers such as ALP and OCN gradually increased during osteogenic differentiation (Fig. 1C and D). Furthermore, the change in miRNA-483-3p expression also gradually increased in hMSCs during osteogenic differentiation (Fig. 1E). The present results suggested that miRNA-483-3p may play an important role in the process of osteogenic differentiation of BMSCs. 
A

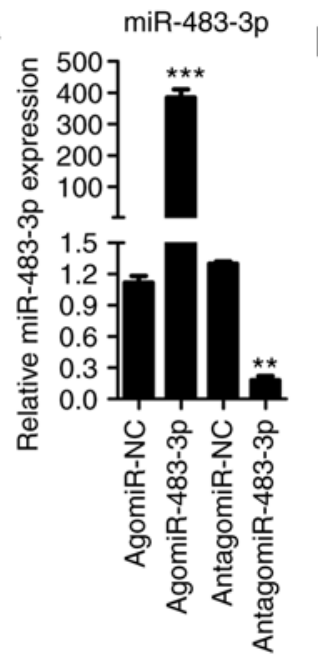

E

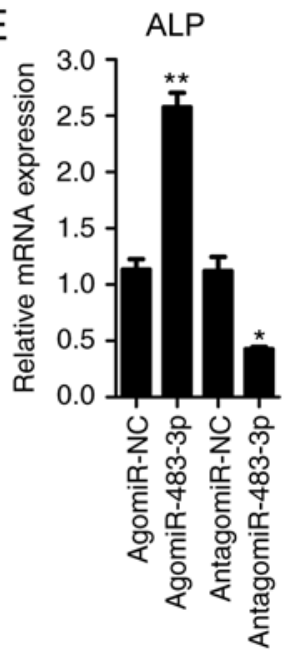

$\mathrm{B}$

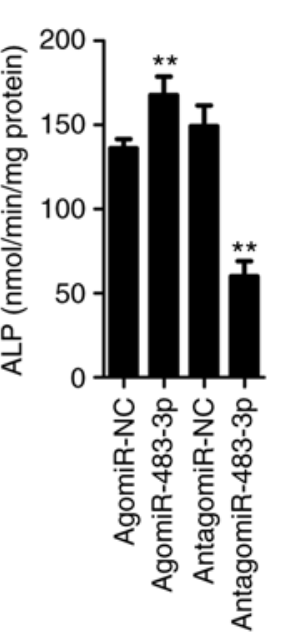

$\mathrm{F}$

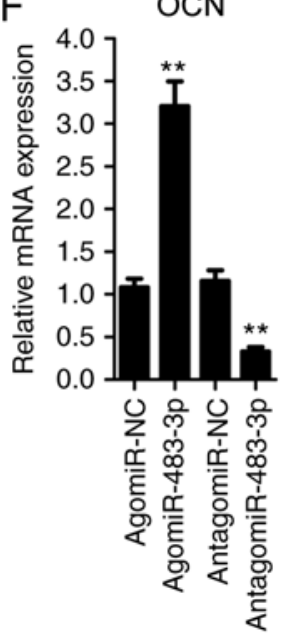

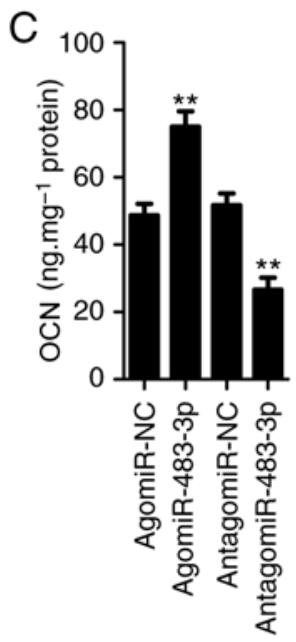
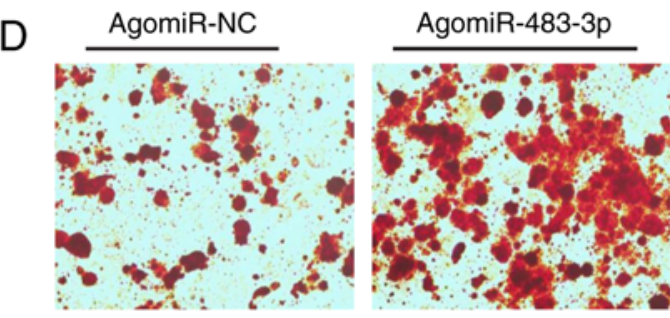

AntagomiR-NC

AntagomiR-483-3p

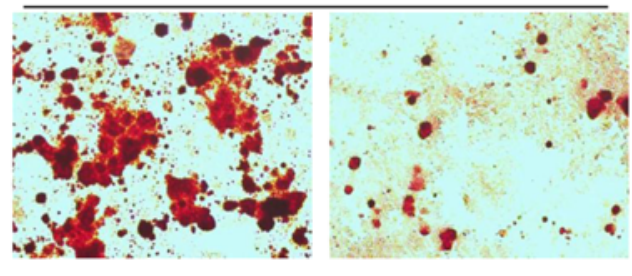

G

G Type 1 collagen

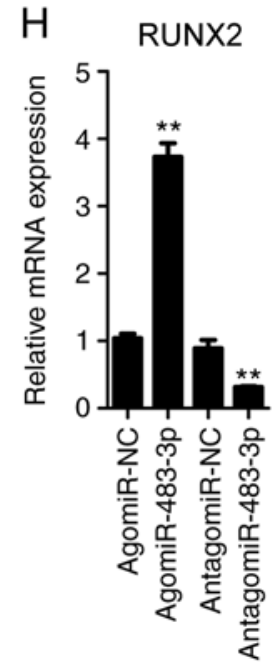

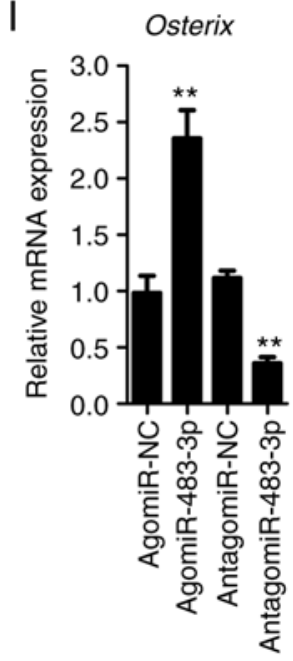

Figure 2. miR-483-3p promotes osteogenic differentiation of BMSCs. (A) RT-qPCR analysis was performed to assess the expression level of miR-483-3p in BMSCs after transfection with agomiR-NC, agomiR-483-3p, antagomiR-NC or antagomiR-483-3p for 48 h. (B) ALP activity and (C) OCN secretion were measured after transfection with agomiR-483-3p, antagomiR-483-3p or NC for $48 \mathrm{~h}$. (D) Alizarin Red staining was used to quantify matrix mineralization in BMSCs after induction of osteoblast differentiation for 21 days. Magnification, x200. RT-qPCR analysis of the mRNA levels of (E) ALP, (F) OCN, (G) Type 1 collagen, (H) RUNX2 and (I) osterix in BMSCs cultured in osteogenic differentiation medium for $48 \mathrm{~h}$. Data are presented as the mean \pm SD. $\mathrm{n}=5$ in each group. ${ }^{*} \mathrm{P}<0.05,{ }^{* *} \mathrm{P}<0.01,{ }^{* * *} \mathrm{P}<0.001$ vs. corresponding NC. BMSCs, bone marrow mesenchymal stem cells; miR, microRNA; ALP, alkaline phosphatase; OCN, osteocalcin; RUNX2, runt-related transcription factor 2; NC, negative control; RT-qPCR, reverse transcription-quantitative PCR.

miR-483-3p promotes osteogenic differentiation of BMSCs. The expression level of miR-483-3p increased during osteogenic differentiation, which suggested that miR-483 may have a role in promoting osteogenic differentiation. To test this hypothesis, BMSCs were transfected with agomiR-483-3p, antagomiR-483-3p or control, and cultured in osteogenic differentiation medium for $48 \mathrm{~h}$. The expression of miR-483-3p was assessed by qPCR (Fig. 2A). ALP activity and OCN secretion, two of the most important osteogenic differentiation markers (4), were identified to be increased in agomiR-483-3p-transfected BMSCs compared with the control group (Fig. 2B and C). By contrast, these effects were reversed following antagomiR-483-3p transfection (Fig. 2B and C). In addition, overexpression of miR-483-3p increased mineralized nodule formation following a21-day incubation in osteogenesis induction medium, as assessed by Alizarin Red staining (Fig. 2D). By contrast, knockdown of miR-483-3p decreased the mineralized nodule formation (Fig. 2D). Moreover, the expression of osteogenic gene markers such as ALP, OCN, Type I collagen, RUNX2 and osterix were also increased following transfection with agomiR-483-3p (Fig. 2E-I). However, the transfection of antagomiR-483-3p decreased the expression levels of these genes (Fig. 2E-I). Collectively, the present results suggested that miR-483-3p promoted osteogenic differentiation of BMSCs.

miR-483-3p directly targets STAT1 to regulate osteogenic differentiation. To understand the molecular mechanisms underlying miR-483-3p promotion of osteogenic differentiation, the potential target genes of miR-483-3p were investigated using the miRNA target prediction tools Targets can and miRanda. Among the putative target genes identified, the present study examined STAT1, which was predicted to be a potential target gene in both databases, and has previously been reported to regulate osteogenic differentiation (21). Sequence analysis suggested that miR-483-3p may target STAT1 by binding to its 3'-UTR in the position 1511-1517 
A

\begin{tabular}{rcccc} 
WT-STAT1-3'-UTR & $5^{\prime}$ & ...CUUCCCAUGUCUCCAGAGGAGUU... & $3^{\prime}$ \\
miR-483-3p & $3^{\prime}$ & UGUUCUGCCCUCCCCUCCUCAC & $5^{\prime}$ \\
MUT-STAT1-3'-UTR & $5^{\prime}$ & ...CUUCCCAUGUCUCCAGUUAAGUU... & $3^{\prime}$ \\
& & & miR-483-3p target site \\
5'-UTR & & STAT1-CDS & 3'-UTR \\
\hline
\end{tabular}

$1511-1517$
B

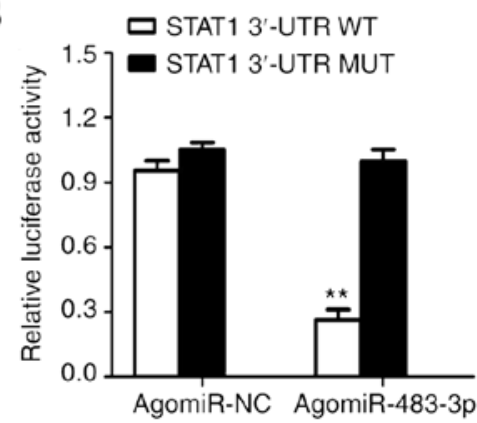

C

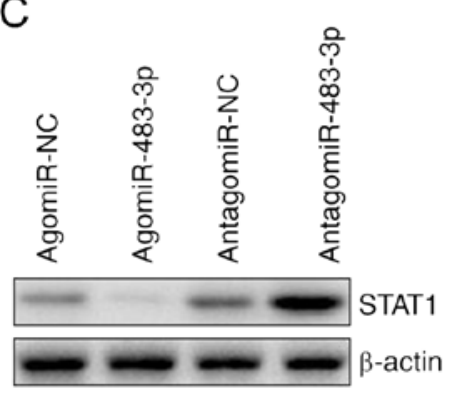

D
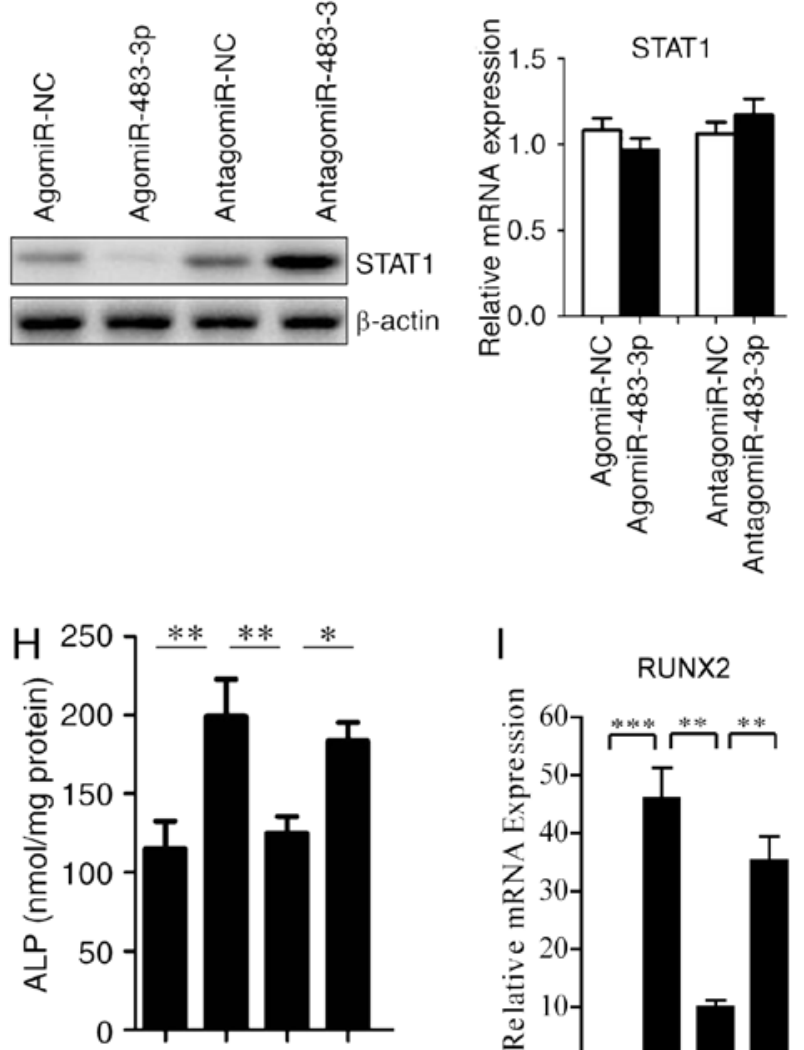

$\begin{array}{rllll}\text { Control } & + & - & - & - \\ \text { RUNX2 } & - & + & + & + \\ \text { STAT1 } & - & - & + & + \\ \text { miR- } & - & - & - & + \\ \text { 483-3P } & & & & \end{array}$
I

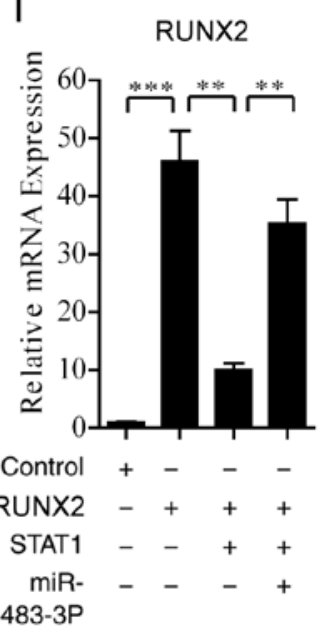

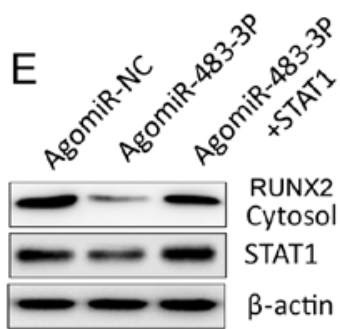

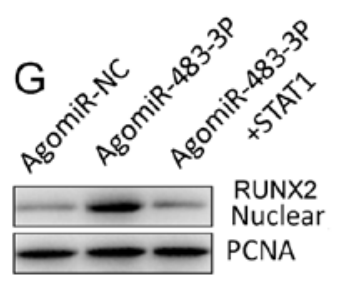

$J$

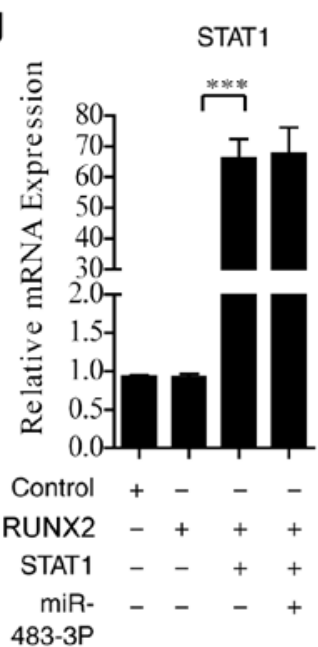

$\mathrm{F}$

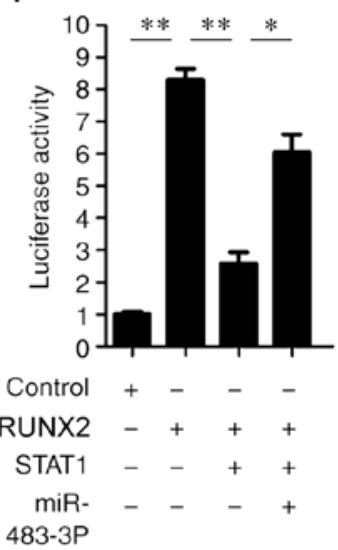

$\mathrm{K}$
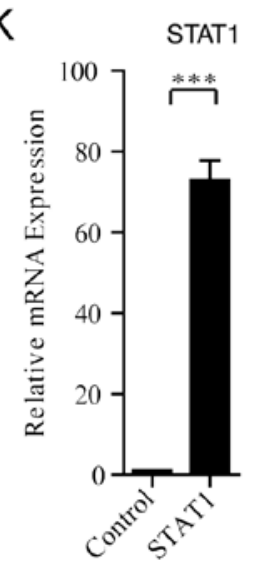

Figure 3. miR-483-3p targets STAT1 to functionally promote osteogenic differentiation. (A) Base pairing of miR-483-3p with STAT1 3'-UTR. Luciferase reporters with the WT-STAT13'-UTR and the MUT-STAT13'-UTR. (B) AgomiR-NC or agomiR-483-3p were co-transfected with WT-STAT13'-UTR or MUT-STAT1 3'-UTRinto BMSCs, and luciferase activity was determined $48 \mathrm{~h}$ after transfection. Renilla activity was used to normalize Firefly luciferase activity. (C) Western blotting and (D) RT-qPCR analysis of the relative expression level of STAT1 after transfection with agomiR-483-3p or antagomiR-483-3p into BMSCs. (E) Western blot analysis of STAT1 and RUNX2 protein expression in the cytoplasm. $\beta$-actin was used as loading control. (F) Luciferase reporter assay of OCN promoter activity in BMSCs after co-transfection with a dual-luciferase reporter plasmid containing the OCN promoter along with RUNX2 plasmid or RUNX2 and STAT1 overexpression vectors. (G) Western blot analysis of RUNX2 protein expression in the nucleus. PCNA was used as loading control. (H) ALP activity in BMSCs was analyzed after transfection with RUNX2 plasmid or RUNX2 and STAT1 plasmids. (I) RUNX2 and (J) STAT1 expression levels were analyzed in the four experimental groups. (K) STAT1 expression level was analyzed following transfection of STAT1 plasmid. Data are presented as the mean $\pm \mathrm{SD} . \mathrm{n}=5$ in each group. ${ }^{*} \mathrm{P}<0.05,{ }^{* *} \mathrm{P}<0.01,{ }^{* * *} \mathrm{P}<0.001$. miR, microRNA; RUNX2, runt-related transcription factor 2 ; OCN, osteocalcin; RT-qPCR, reverse transcription-quantitative PCR; UTR, untranslated region; BMSCs, bone marrow mesenchymal stem cells; CDS, coding sequence; MUT, mutant; WT, wild-type; PCNA, proliferating cell nuclear antigen; ALP, alkaline phosphatase; NC, negative control.

(Fig. 3A). To examine whether miR-483-3p can directly bind the 3'-UTR of STAT1, a luciferase reporter plasmid containing the 3'-UTR of STAT1 and a mutated luciferase reporter plasmid were constructed (Fig. 3A). Wild-type and mutant 

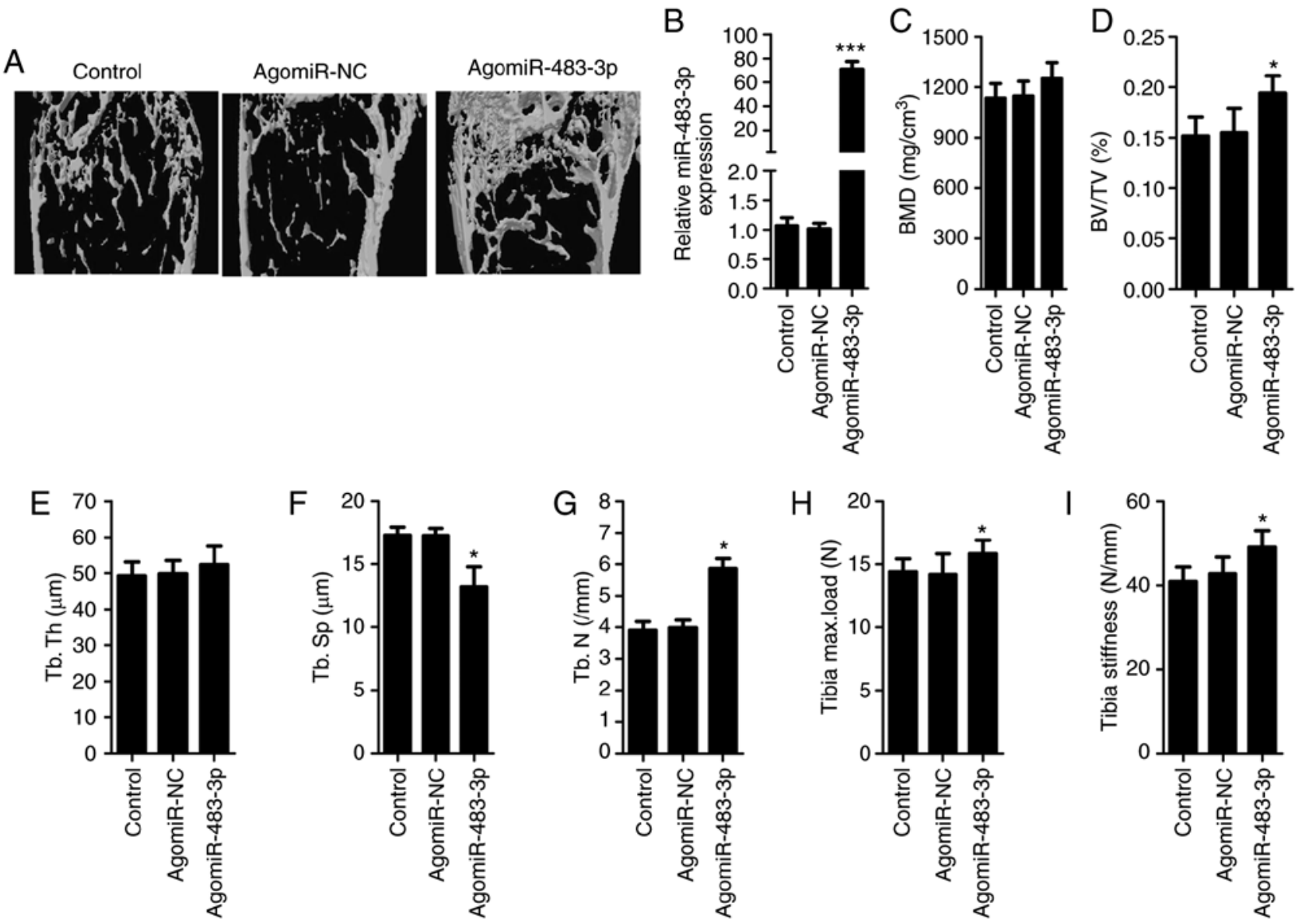

Figure 4. miR-483-3p regulates bone formation in vivo. BMSC-specific aptamer-agomiR-483-3p, aptamer-agomiR-NC or PBS was periosteally injected into 12-month-old mice twice per month for 3 months. (A) Effect of BMSC-specific aptamer-agomiR-483-3p in aged mice as determined by microCT examination. (B) RT-qPCR analysis of the expression level of miR-483-3P in bone. Quantitative microCT analysis of femoral histomorphometric parameters, including (C) BMD, (D) BV/TV, (E) Tb.Th, (F) Tb.Sp and (G) Tb.N. Three-point bending measurement of tibia (H) maximum load and (I) stiffness. Data are presented as the mean \pm SD. $n=6$ in each group. ${ }^{*} \mathrm{P}<0.05,{ }^{* * * *} \mathrm{P}<0.001$ vs. control. NC, negative control; miR, microRNA; BMSCs, bone marrow mesenchymal stem cells; BMD, bone mineral density; BV/TV, bone volume/tissue volume; Tb.Th, trabecular thickness; Tb.Sp, trabecular spacing; Tb.N, trabecular number; microCT, microcomputed tomography.

STAT1 luciferase expression vectors were then transfected into BMSCS cells along with agomiR-483-3p. Transfection with agomiR-483-3p suppressed the luciferase activity of the wild-type STAT1 3'-UTR, but exhibited no effects on the mutated version of STAT1 3'-UTR (Fig. 3B). Moreover, overexpression of miR-483-3p downregulated endogenous STAT1 protein expression, whereas inhibition of miR-483-3p increased STAT1 protein expression levels (Fig. 3C). However, STAT1 mRNA levels were not affected by transfection with agomiR-483-3p or antagomiR-483-3p (Fig. 3D). The present results suggested that miR-483-3p targetedSTAT1 by binding to its 3'-UTR and affectedSTAT1 expression at the post-transcriptional level.

A previous study reported that STAT1 inhibits osteoblast differentiation by inhibiting RUNX2 transcriptional activity and RUNX2 nuclear translocation, which has been reported to have a key role in osteogenic differentiation and bone formation (21). In the present study, miR-483-3p was identified to downregulate STAT1 expression, and it was hypothesized that miR-483-3p may promote osteogenic differentiation of BMSCs by downregulating STAT1 and enhancing RUNX2 transcriptional activity and nuclear translocation. Interestingly, a previous study reported that overexpression of STAT1 significantly inhibited RUNX2 nuclear translocation and osteoblast differentiation mediated by miR-194 (15). In the present study, overexpression of miR-483-3p was identified to increase the nuclear translocation of RUNX2, but this effect was suppressed by transfection with STAT1 overexpression plasmid (Fig. 3E and G). Next, to study the effects of miR-483-3p on RUNX2 transcriptional activity, a reporter plasmid containing the $\mathrm{OCN}$ promoter was transfected into BMSCs along with RUNX2 vector, STAT1 plasmid, and/or agomiR-483-3p. Overexpression of RUNX2 significantly increased RUNX2-mediated luciferase activity in a plasmid containing the $\mathrm{OCN}$ promoter, whereas co-transfection with STAT1 overexpression plasmid suppressed this effect. Interestingly, RUNX2-mediated luciferase activity was rescued by co-transfection with agomiR-483-3p (Fig. 3F). The present results suggested that miR-483-3p promoted osteogenic differentiation of BMSCs by increasing RUNX2 nuclear translocation and RUNX2 transcriptional activity via themiR-483-3p/STAT1/RUNX2 axis.

To further investigate this hypothesis, RUNX2, STAT1 or agomiR-483-3p were transfected into BMSCs to investigate their effects on ALP activity. The present results suggested that ALP activity was significantly increased in the RUNX2 
overexpression plasmid transfection group, whereas this effect was inhibited following STAT1 overexpression. Notably, ALP activity was rescued following agomiR-483-3p co-transfection (Fig. 3H). Additionally, the transfection efficiencies of RUNX2 and STAT1 overexpression plasmids were examined (Fig. 3I-K). Collectively, the present results suggested that $\mathrm{miR}-483-3 \mathrm{p}$ regulated osteogenic differentiation via themiR-483-3p/STAT1/RUNX2 axis.

$m i R-483-3 p$ regulates bone formation in vivo. In order to examine the effects of miR-483-3p in vivo, BMSC-specific aptamer-agomiR-NC,BMSC-specific aptamer-agomiR-483-3P or PBS was injected into the femoral bone marrow cavity of 12-month-old mice twice per month for 3 months $(n=6$ in each group). After 3 months, miR-483-3p mRNA levels were measured by RT-qPCR in mice bone tissues. The results showed that the levels of miR-483-3p were significantly increased in bone after injection of BMSC-targeted aptamer-agomiR-483-3p (Fig. 4B). Moreover, these mice exhibited significantly higher bone density, trabecular bone volume per tissue volume, trabecular thickness, and tibia maximum load and stiffness, and lower trabecular separation compared with vehicle-treated mice (Fig. 4). Furthermore, the number of osteoblasts on the bone surface was significantly increased in mice treated with agomiR-483-3p compared with controls (Fig. 4G). The present results suggested that overexpression of miR-483-3p increased bone formation in aged mice.

\section{Discussion}

Numerous previous studies identified that miRNAs serve an important regulatory role in bone biology, including osteogenic differentiation and bone formation $(4,5)$. In the present study, miR-438-3p expression was identified to gradually increase during osteogenic differentiation. In addition, miR-438-3p promoted osteogenic differentiation of BMSCs by downregulating STAT1 and enhancing the transcriptional activity of RUNX2. Present in vivo experiments suggested that increasing miR-483-3p levels using a BMSC-specific delivery system promoted bone formation in aged mice.

miR-483-3p has been widely investigated in various cellular processes and cell types. Specifically, miR-483-3p has been reported to be involved in regulating apoptosis, proliferation, diabetes-associated heart diseases and inhibition of adipocyte differentiation $(22,23)$. However, the role of miR-483-3p in regulating osteogenic differentiation of BMSCs remains unclear. In the present study, a novel role of miR-483-3p and its underlying mechanism were identified, and $\mathrm{miR}-483-3 \mathrm{p}$ was found to be involved in regulating the osteogenic differentiation of BMSCs by targeting STAT1.

STAT1 was first identified as a signaling molecule in the interferon signaling pathway $(24,25)$. A previous study identified that STAT1 is also involved in osteogenic differentiation and bone formation (26). STAT1 mutant mice exhibit increased bone formation and bone mass, although they also present increased osteoclasts and bone resorption (21). In addition, a previous study demonstrated that STAT1 directly interacts and inhibits RUNX2, a transcription factor with a key role in osteoblast differentiation and bone formation, suppressing the nuclear localization and transcriptional activity of RUNX2 $(15,27)$. These previous findings suggested that STAT1 functions as a potent osteogenic inhibitor able to suppress bone formation in vivo $(15,27)$. A previous study suggested that downregulation of STAT1 could be used as a potential strategy to improve bone formation or osteogenic differentiation (21). The present results suggested that the change in miR-483-3p levels resulted in an alteration in the amount of STAT1 protein. In addition, overexpression of STAT1 can abolish the positive effect of RUNX2 on ALP activity in BMSCs, which is rescued by agomiR-483-3p transfection. Collectively, the present results suggested that STAT1 was a functional target of $\mathrm{miR}-483-3 \mathrm{p}$ and that increasing the expression level ofmiR-483-3p may be a potential strategy for promoting osteogenic differentiation.

Due to the increase in the aging population, osteoporosis has become a major public health problem (28). Intermittent injections of parathyroid hormone 1-38 is a common treatment approach for osteoporosis, but this approach is limited to a 2-year time period and has some potential side effects, such as excessive bone resorption $(29,30)$. Novel safe anabolic drugs that increase bone formation are thus required for treating this disease. Previous studies demonstrated that intra-bone marrow injection of agomiR or antagomiR with a BMSC-specific aptamer increased BMSC differentiation and bone formation, suggesting that this approach may represent a potential a strategy to treat osteoporosis $(4,31)$. In the present study, a BMSC-specific aptamer was used to deliver agomiR-483-3p into mouse BMSCs via intra-bone marrow injections. The present results suggested that overexpression of miR-483-3p increased bone formation in aged mice. The present results support the aptamer-mediated modulation of miRNA expression levels in BMSCs as a new strategy to treat senile osteoporosis.

Collectively, the present findings suggested that miR-483-3p promoted osteogenic differentiation of BMSCs by targeting STAT1 and enhancing the transcriptional activity of RUNX2, identifying a new role for miR-483-3p and suggesting a novel potential therapeutic target to treat osteoporosis.

\section{Acknowledgements}

Not applicable.

\section{Funding}

The present study was supported by The National Natural Science Foundation of China (grant nos. 81700785, 81500686, 81770877 and 81670809).

\section{Availability of data and materials}

The datasets used and/or analyzed during the current study are available from the corresponding author on reasonable request.

\section{Authors' contributions}

YX performed the experiments. WX designed the study. QG and TJJ analyzed and interpreted the data. YY, LY and GWW 
performed the statistical analysis and drafted the manuscript All authors read and approved the final manuscript.

\section{Ethics approval and consent to participate}

Human mesenchymal stem cells were isolated from the bone marrow of healthy donors. The procedure was approved by The Ethics Committee of the Xiangya Hospital of Central South University. All animal experimental procedures were approved by The Animal Care and Use Committee of the Xiangya Hospital of Central South University.

\section{Patient consent for publication}

All patients provided written informed consent.

\section{Competing interests}

The authors declare that they have no competing interests.

\section{References}

1. Krane SM: Identifying genes that regulate bone remodeling as potential therapeutic targets. J Exp Med 201: 841-843, 2005.

2. Pittenger MF, Mackay AM, Beck SC, Jaiswal RK, Douglas R, Mosca JD, Moorman MA, Simonetti DW, Craig S and Marshak DR: Multilineage potential of adult human mesenchymal stem cells. Science 284: 143-147, 1999.

3. Crane JL and Cao X: Bone marrow mesenchymal stem cells and TGF- $\beta$ signaling in bone remodeling. J Clin Invest 124: 466-472, 2014.

4. Li CJ, Cheng P, Liang MK, Chen YS, Lu Q, Wang JY, Xia ZY, Zhou HD, Cao X, Xie H, et al: MicroRNA-188 regulates age-related switch between osteoblast and adipocyte differentiation. J Clin Invest 125: 1509-1522, 2015.

5. Khalaj M, Woolthuis CM, Hu W, Durham BH, Chu SH Qamar S, Armstrong SA and Park CY: miR-99 regulates normal and malignant hematopoietic stem cell self-renewal. J Exp Med: Jul 21, 2017 (Epub ahead of print). jem.20161595, 2017. doi: 10.1084/jem.20161595.

6. Edmonds MD, Boyd KL, Moyo T, Mitra R, Duszynski R, Arrate MP, Chen X, Zhao Z, Blackwell TS, Andl T and Eischen CM: MicroRNA-31 initiates lung tumorigenesis and promotes mutant KRAS-driven lung cancer. J Clin Invest 126: 349-364, 2016.

7. Mori MA, Thomou T, Boucher J, Lee KY, Lallukka S, Kim JK, Torriani M, Yki-Järvinen H, Grinspoon SK, Cypess AM and Kahn CR: Altered miRNA processing disrupts brown/white adipocyte determination and associates with lipodystrophy. J Clin Invest 124: 3339-3351, 2014.

8. Zhou X, Jeker LT, Fife BT, Zhu S, Anderson MS, McManus MT and Bluestone JA: Selective miRNA disruption in T reg cells leads to uncontrolled autoimmunity. J Exp Med 205: 1983-1991, 2008.

9. Li H, Xie H, Liu W, Hu R, Huang B, Tan YF, Xu K, Sheng ZF, Zhou HD, Wu XP and Luo XH: A novel microRNA targeting HDAC5 regulates osteoblast differentiation in mice and contributes to primary osteoporosis in humans. J Clin Invest 119: 3666-3677, 2009.

10. Li Z, Hassan MQ, Volinia S, van Wijnen AJ, Stein JL, Croce CM, Lian JB and Stein GS: A microRNA signature for a BMP2-induced osteoblast lineage commitment program. Proc Natl Acad Sci USA 105: 13906-13911, 2008

11. Gu H, Xu J, Huang Z, Wu L, Zhou K, Zhang Y, Chen J, Xia J and Yin $X$ : Identification and differential expression of microRNAs in 1,25-dihydroxy vitamin D3-induced osteogenic differentiation of human adipose-derived mesenchymal stem cells. Am J Transl Res 11: 4856-4871, 2017.

12. Maruyama K, Uematsu S, Kondo T, Takeuchi O, Martino MM, Kawasaki T and Akira S: Strawberry notch homologue 2 regulates osteoclast fusion by enhancing the expression of DC-STAMP. J Exp Med 210: 1947-1960, 2013.

13. Livak KJ and Schmittgen TD: Analysis of relative gene expression data using real-time quantitative PCR and the 2(-Delta Delta C(T)) method. Methods 25: 402-408, 2001.
14. Javed A, Gutierrez S, Montecino M, van Wijnen AJ, Stein JL, Stein GS and Lian JB: Multiple Cbfa/AML sites in the rat osteocalcin promoter are required for basal and vitamin D-responsive transcription and contribute to chromatin organization. Mol Cell Biol 19: 7491-7500, 1999.

15. Li J, He X, Wei W and Zhou X: MicroRNA-194 promotes osteoblast differentiation via downregulating STAT1. Biochem Biophys Res Commun 460: 482-488, 2015.

16. Zou W, Greenblatt MB, Brady N, Lotinun S, Zhai B, de Rivera H, Singh A, Sun J, Gygi SP, Baron R, et al: The microtubule-associated protein DCAMKL1 regulates osteoblast function via repression of RUNX2. J Exp Med 210: 1793-1806, 2013.

17. Xiao WZ, Gu XC, Hu B, Liu XW, Zi Y and Li M: Role of microRNA-129-5p in osteoblast differentiation from bone marrow mesenchymal stem cells. Cell Mol Biol (Noisy-le-grand) 62: 95-99, 2016.

18. Grünhagen J, Bhushan R, Degenkolbe E, Jäger M, Knaus P, Mundlos S, Robinson PN and Ott CE: miR-497 approximately 195 cluster microRNAs regulate osteoblast differentiation by targeting BMP signaling. J Bone Miner Res 30: 796-808, 2015.

19. Bertero T, Gastaldi C, Bourget-Ponzio I, Imbert V, Loubat A, Selva E, Busca R, Mari B, Hofman P, Barbry P, et al: miR-483-3p controls proliferation in wounded epithelial cells. FASEB J 25: 3092-3105, 2011

20. Lupini L, Pepe F, Ferracin M, Braconi C, Callegari E, Pagotto S, Spizzo R, Zagatti B, Lanuti P, Fornari F, et al: Over-expression of the miR-483-3p overcomes the miR-145/TP53 pro-apoptotic loop in hepatocellular carcinoma. Oncotarget 7: 31361-31371, 2016.

21. Kim S, Koga T, Isobe M, Kern BE, Yokochi T, Chin YE, Karsenty G, Taniguchi T and Takayanagi H: Statl functions as a cytoplasmic attenuator of RUNX2 in the transcriptional program of osteoblast differentiation. Genes Dev 17: 1979-1991, 2003.

22. Bertero T, Bourget-Ponzio I, Puissant A, Loubat A, Mari B, Meneguzzi G, Auberger P, Barbry P, Ponzio G and Rezzonico R: Tumor suppressor function of miR-483-3p on squamous cell carcinomas due to its pro-apoptotic properties. Cell Cycle 12: 2183-2193, 2013.

23. Ferland-McCollough D, Fernandez-Twinn DS, Cannell IG David H, Warner M, Vaag AA, Bork-Jensen J, Brøns C, Gant TW, Willis AE, et al: Programming of adipose tissue miR-483-3p and GDF-3 expression by maternal diet in type 2 diabetes. Cell Death Differ 19: 1003-1012, 2012.

24. Meraz MA, White JM, Sheehan KC,Bach EA, Rodig SJ,Dighe AS, Kaplan DH, Riley JK, Greenlund AC, Campbell D, et al: Targeted disruption of the Statl gene in mice reveals unexpected physiologic specificity in the JAK-STAT signaling pathway. Cell 84: 431-442, 1996.

25. Huang Z, Richmond TD, Muntean AG, Barber DL, Weiss MJ and Crispino JD: STAT1 promotes megakaryopoiesis downstream of GATA-1 in mice. J Clin Invest 117: 3890-3899, 2007.

26. Takayanagi H, Ogasawara K, Hida S, Chiba T, Murata S, Sato K, Takaoka A, Yokochi T, Oda H, Tanaka K, et al: T-cell-mediated regulation of osteoclastogenesis by signalling cross-talk between RANKL and IFN-gamma. Nature 408: 600-605, 2000.

27. Tajima K, Takaishi H, Takito J, Tohmonda T, Yoda M, Ota N, Kosaki N, Matsumoto M, Ikegami H, Nakamura T, et al: Inhibition of STAT1 accelerates bone fracture healing. J Orthop Res 28: 937-941, 2010

28. Slemenda C, Longcope C, Peacock M, Hui S and Johnston CC: Sex steroids, bone mass, and bone loss. A prospective study of pre-, peri-, and postmenopausal women. J Clin Invest 97: 14-21, 1996.

29. Lane NE, Sanchez S, Modin GW, Genant HK, Pierini E and Arnaud CD: Parathyroid hormone treatment can reverse corticosteroid-induced osteoporosis. Results of a randomized controlled clinical trial. J Clin Invest 102: 1627-1633, 1998.

30. Balani DH, Ono N and Kronenberg HM: Parathyroid hormone regulates fates of murine osteoblast precursors in vivo. J Clin Invest 127: 3327-3338, 2017.

31. Wang X, Guo B, Li Q, Peng J, Yang Z, Wang A, Li D, Hou Z, Lv K, Kan G, et al: miR-214 targets ATF4 to inhibit bone formation. Nat Med 19: 93-100, 2013.

This work is licensed under a Creative Commons Attribution-NonCommercial-NoDerivatives 4.0 International (CC BY-NC-ND 4.0) License. 